\title{
ESTIMATING THE TECHNICAL EFFICIENCY OF VEGETABLE PRODUCERS AT THE AIR CONDITIONED AND NON-AIR CONDITIONED GREEN HOUSES USING STOCHASTIC FRONTIER FUNCTION
}

(Received: 5.2.2009)

\author{
By \\ Kh. N. Alrwis \\ Faculty of Agriculture and Food Sciences, Agricultural Economics Department, King Saoud \\ University, Kingdom of Saudi Arabia
}

\begin{abstract}
The study aimed at estimating the technical efficiency of vegetable producing airconditioned and non-air conditioned green houses. The study also aimed at examining the effect of the size of green houses on their technical efficiency within the KSA. The study relied on basic data collected from enquiry forms which were applied to stochastic sample of air conditioned and non-air conditioned green houses, representing all regions of the KSA. The sample included 62 projects of non-air conditioned projects and 22 projects of air conditioned ones in the summer and autumn of 2007. The study made use of stochastic frontier functions of production according to actual hypotheses about how to deal with the stochastic errors.

Based on standard estimates of the stochastic frontier function of the non-air conditioned green houses, it appears that the increase in any of the greenhouse areas, the number of employees or the variable costs of $10 \%$, leads to an increase in the production of vegetables by a percentage of $9 \%, 0.7 \%$ and $0.2 \%$, respectively. On the other hand, the increase in the fixed cost, by a percentage of $10 \%$, leads to a decrease in the vegetable production by $0.6 \%$. The estimated technical efficiency by use of the stochastic frontier function for the non-air conditioned green houses ranged from a minimum of $0.10 \%$, a maximum of $0.97 \%$ and an average of $0.46 \%$. As for the air conditioned green houses, it appears that an increase in any of the fixed costs, the areas of the greenhouse and the variable costs by $10 \%$, leads to an increase of vegetable production at percentages of $10.6 \%, 11.1 \%$ and $2.9 \%$, respectively. In addition, the estimated technical efficiency of the stochastic frontier function of air conditioned green houses ranged from a minimum of $0.13 \%$, a maximum of $0.99 \%$ and an average of 0.59 .

The study recommended to upgrade the administrative efficiency of the green house managers, in order to increase production of vegetables of the air conditioned and non-air conditioned green houses by a percentage of 50.4\% and 41.4 , respectively, without any increase in the economic resources. Furthermore, the study recommended to expand the green house projects for producing vegetables since they represent a developed technical approach to increase the production of the vegetables in both quantity and quality, and helps to produce vegetables in other seasons. Accordingly, the fluctuations in prices can be eliminated. In addition, green houses rationalize the consumption of water; they save about $60 \%$ of water used in open air agriculture. This comes in line with the water security standards of the Kingdom of Saudi Arabia.
\end{abstract}

Key words: green house, stochastic frontier function, vegetable producers. 


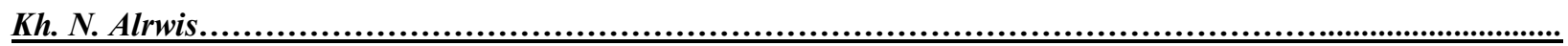

\title{
تقدير الكفاعة التقنية لمنتجي الخضر بالبيوت المحمية المكيفة و غير المكيفة \\ باستخذام الدالة المجالية العشوائية
}

\author{
خالد بن نهار الرويس
}

قسم الاقتصاد الزراعي_ كلية علوم الأغذية و الزراعة - جامعة الملك سعود- المملكة العربية السعودية

\section{ملخص الم المبر}

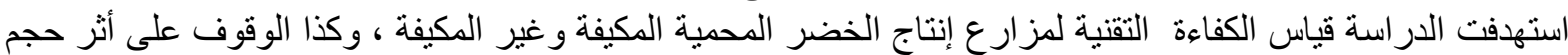

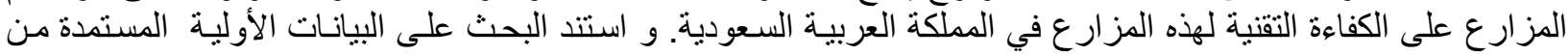

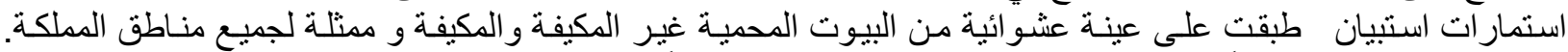

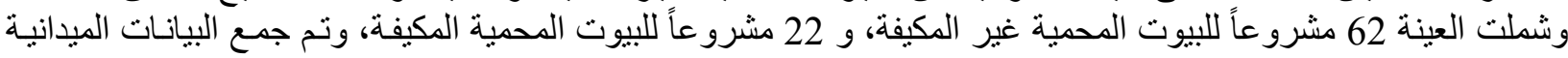

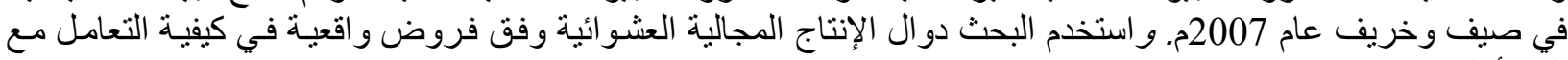

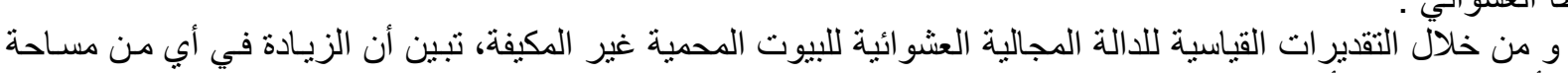
الخطأ العشو ائي .

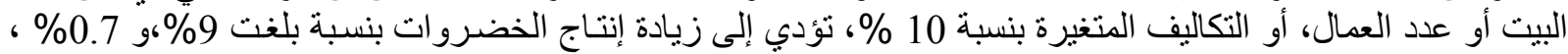

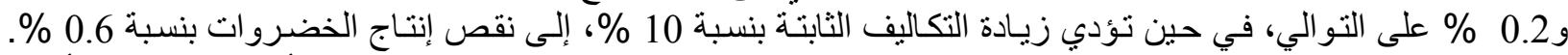

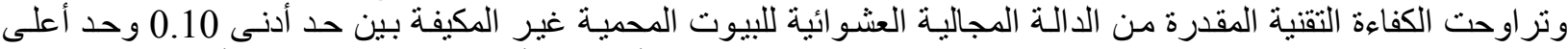

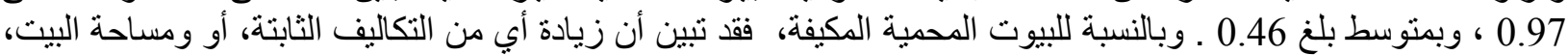

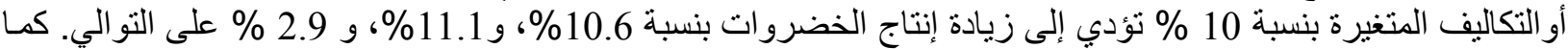

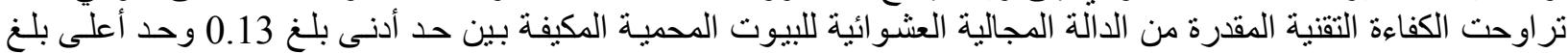

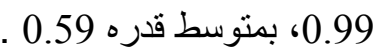

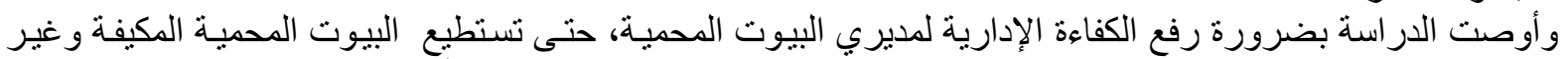

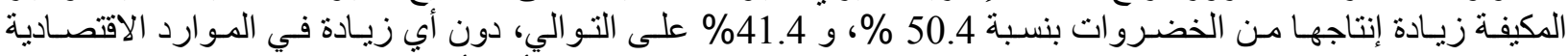

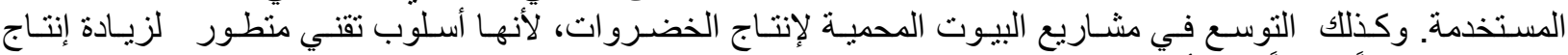

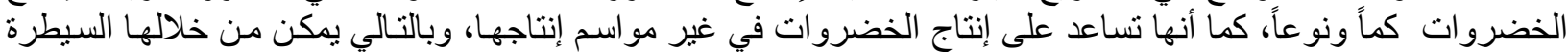

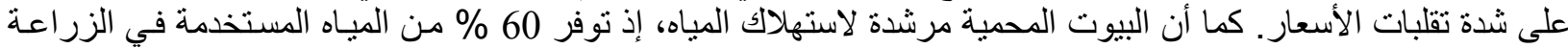
المكشوفة، و هذا يتفق مع اعتبار ات الأمن المائي في المملكة العربية السعودية.

إنتاج تلـك المنـاطق نحو 88.1\%، في حين لاتزيد

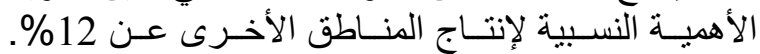

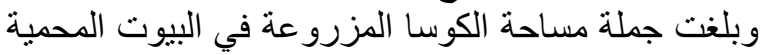

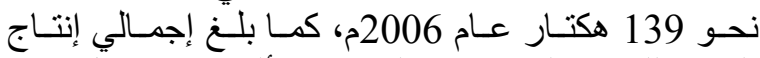

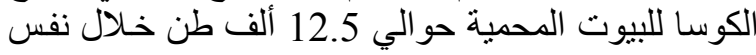

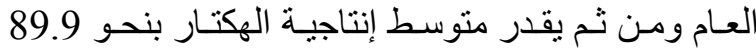
طن/هكتار على مستوى المملكة. يتركز إنتاج الكوسـا في

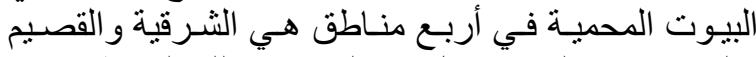

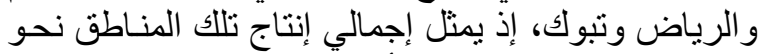

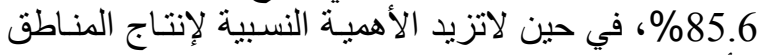

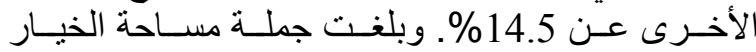

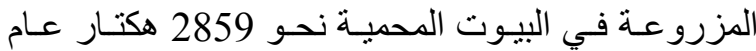

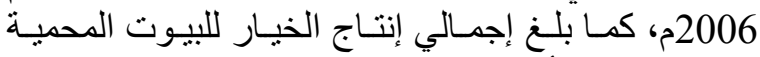

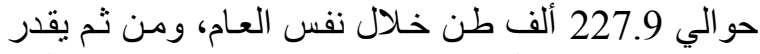

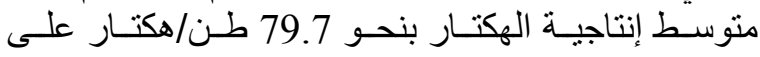

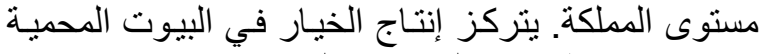

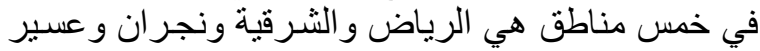

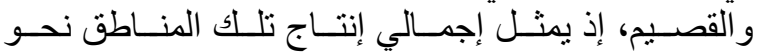

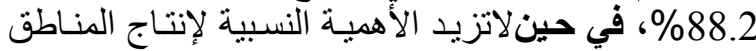

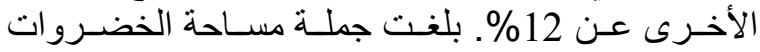
الأخرى المزروعة في البيوت المحمية نحو

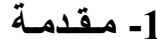

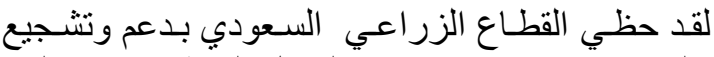

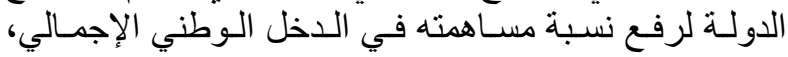

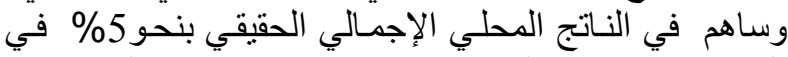

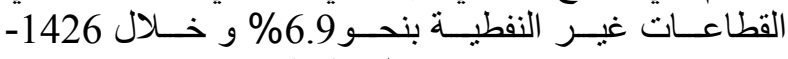

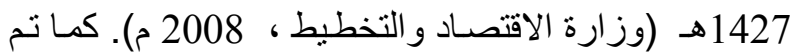

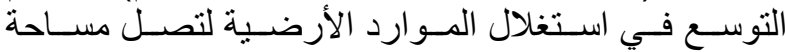

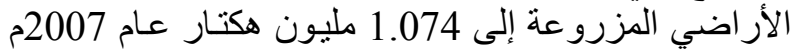

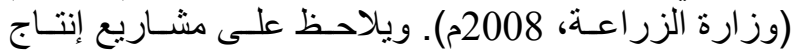

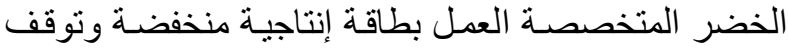

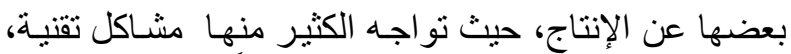

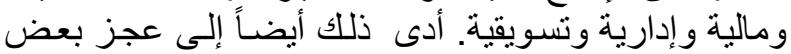

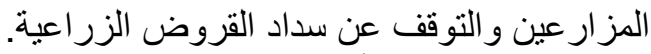

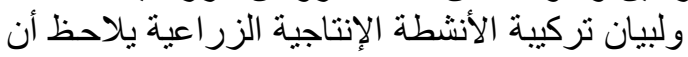

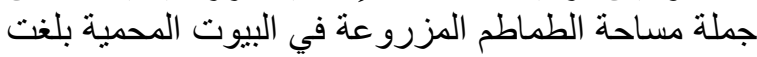

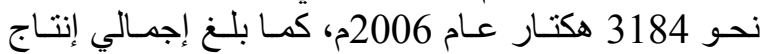

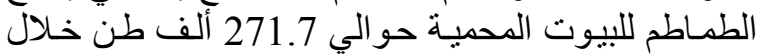

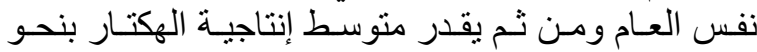

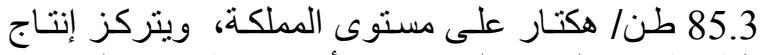

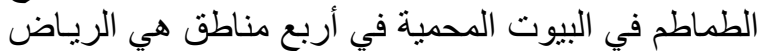
و الثرقية والقصيم و عسير ، إذ يمثل إجمالى الى 


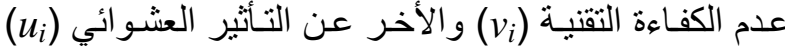

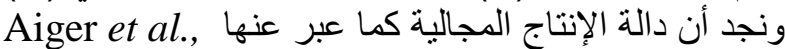

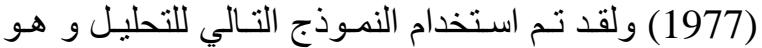

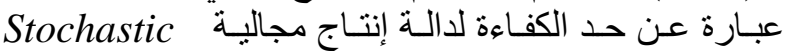
Cobb- مـن نـو frontier production function :: Douglas

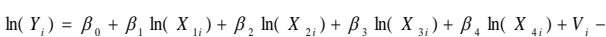

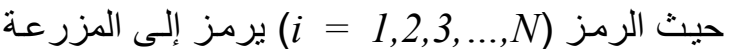

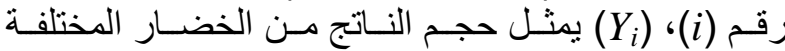

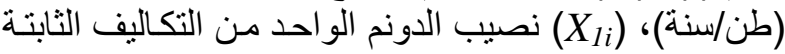

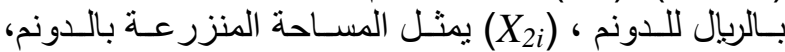

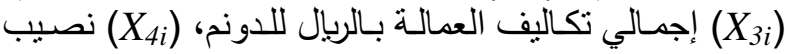

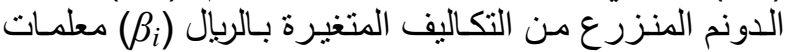

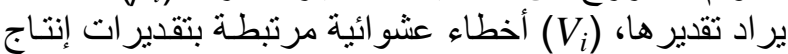

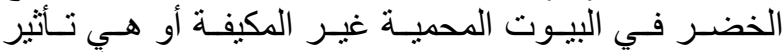

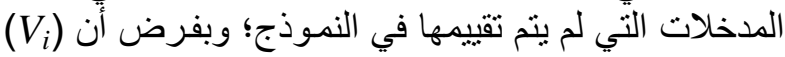

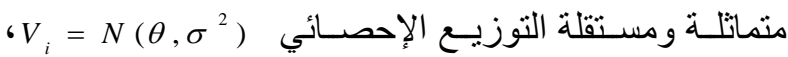

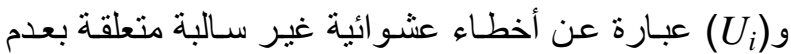

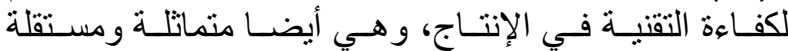
التوزيع بمتوسط (Battese,1998) ( $M_{1}=\delta_{0}+\delta_{1} Z_{2 i}+\delta_{2} Z_{2 i}+\delta_{3} Z_{3 i}$

حيث: Z: $Z_{1 i}$

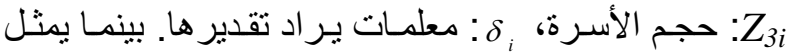

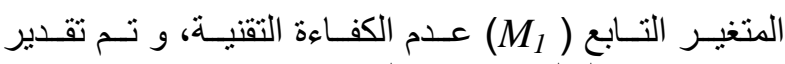
Forntier معاملات هذه الدالة باستخام الحزمة الإحصائية . Coelli,1996) 4.1

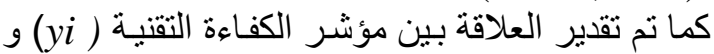
ذات المتغيرات الاجتماعية السابقة على النحو التالي: $y i=\delta_{0}+\delta_{1} Z_{1 i}+\delta_{2} Z_{2 i}+\delta_{3} Z_{3 i}$

مع الأخذ في الاعتبار أن مؤشر الكفاءة التقنية تنتراوح

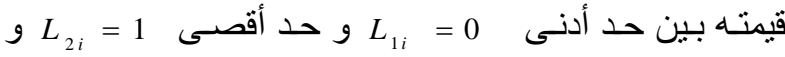

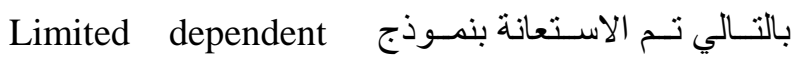
variable, truncated from both sides (Maddala,1987)

$$
\begin{aligned}
& \boldsymbol{Y}^{*}=\delta_{i} \boldsymbol{z}_{j_{i}}+\boldsymbol{u}_{i} \\
& y_{i}=L_{1 i} \quad \text { if } \quad y_{i}<L_{1 i} \\
& y_{i}=Y^{*} \quad \text { if } \quad L_{1 i} \leq y_{i} \leq L_{2 i} \\
& y_{i}=L_{2 i} \text { if } y_{i}>L_{2 i} \\
& \text { وتم تقدير معاملات الدالة باستخدام الحزمة الإحصائية }
\end{aligned}
$$
.NLOGIT Ver.3.0 (Greene, 2003)

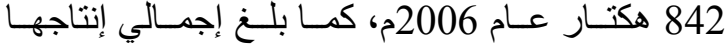
حوالي 70.4 ألف طن خلف خلال نفس العام، ومن ثُم يقدر

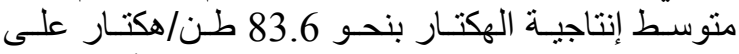
مستوى المملكة. يتركز إنتاج الخضروات النيات الأخرى في

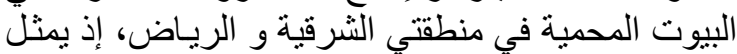

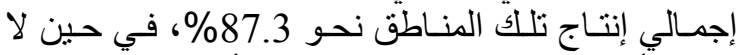

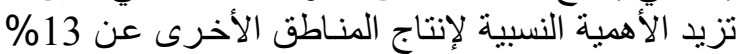

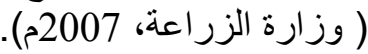

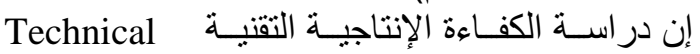
efficiency

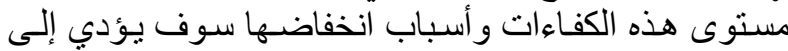

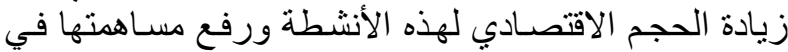
الناتج المحلى وذلك عن طريق رفع مستوى الكفاءة النتنية

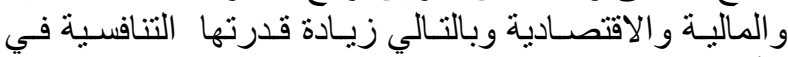

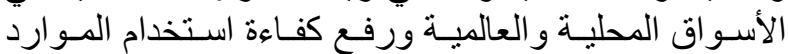
الاقتصادية في القطاع الزراعي العي لتقليل الهدر الاقتصـادي في هذا القطاع المهم.

\section{2- 2- هدف البحث}

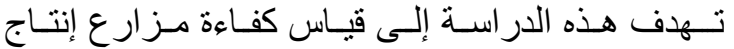
الخضر المتخصصة (البيوت المحمية) الككيفة و غير الكيلة الكيفة

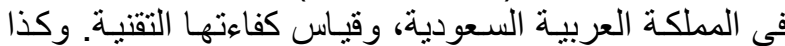
الوقوف على أثر حجم المز ارد على على الكفاءة الثقنية

-1-2 - مصادر البيانات وأسلوب التحليل التيل

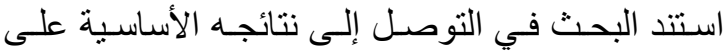

البيانـات الأوليـة data

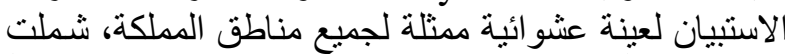
62 مشروعاً للبيوت المحمية غير المكيفة، و 22 مشروعاً

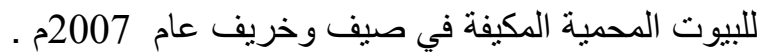

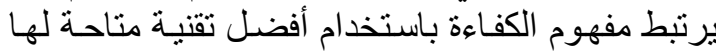

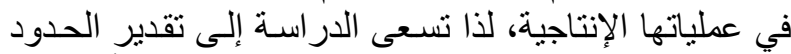

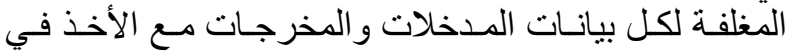
الاعتبار أن المشاهدات التي تقع على الحدود يمكن وصفئا

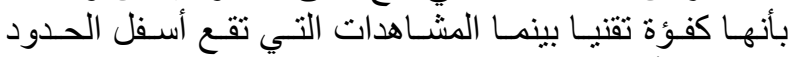
توصف بأنها غير كفؤة تقنيا Technically inefficient واستخدم البحث دوال الإنتاج المجالية العشو ائية التي تتميز

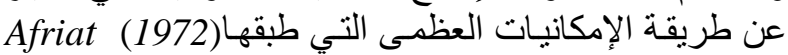

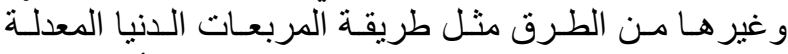
Corrected OLS Stochastic noise بفرضية تقنية الإتناج وفرضية عدم الكفاءة بمكن تطبيقها في

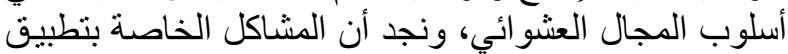

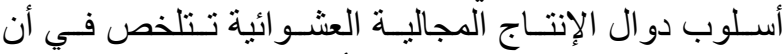
اختيار شكل التوزيع الخاص بتأثئير عدم الكفاءة قد يكون

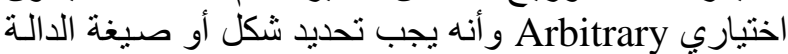

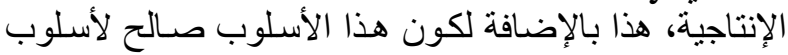
تكنولوجي واحد للإنتاج (Coelli et al.,1998) يمكن تقدير الدالة الإنتاجيـة من بيانات مقطعية، حيث يعبر الخطأ العشوائي للاالة عن مكونين أحدهما يعبر عن 
ومن خلال مستويات المدخلات وطبيعة التقنية المستخدمة

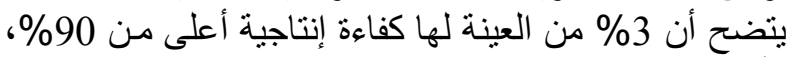

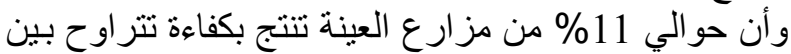

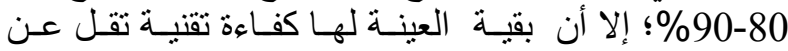

$\% 80$

2-3-30 الكفاعة التقتية لمشاريع البيوت المحمية المكيفة

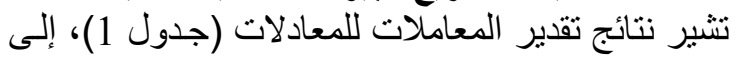

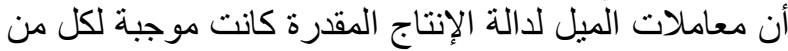

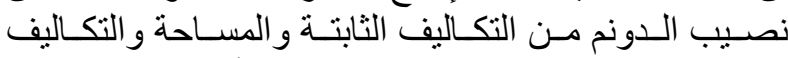

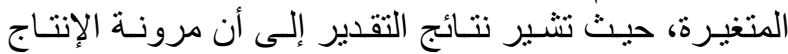

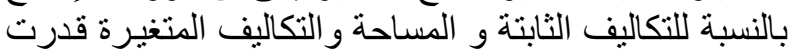

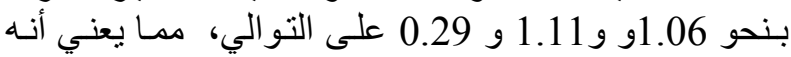

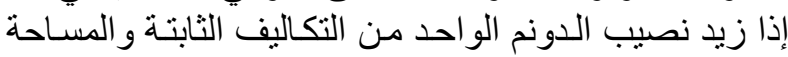

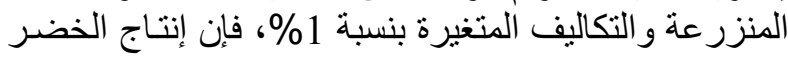

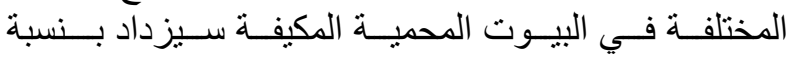
10\% 11 \% و و 0.02\% على التو الي. كذلك قدرت المرونة

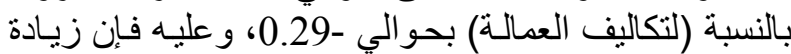

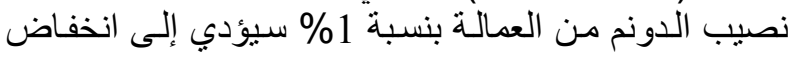

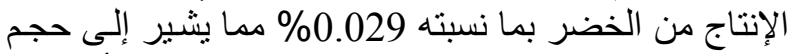

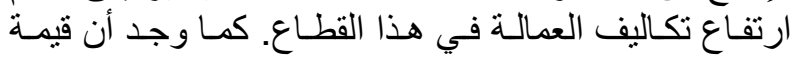
إحصـائية Log Likelihood تسـاوي 18.002480 بينمـا فيـا القيمة المقابلة لـ (OLS) تساوي 28.465709 من هنا يمكن

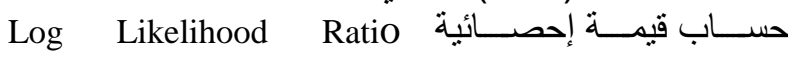
$(L R=-2(28.465709-18.002480)=20.9265)$

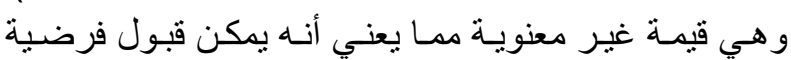

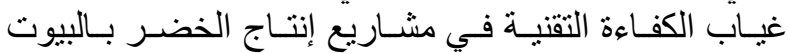

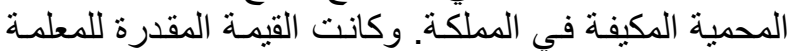

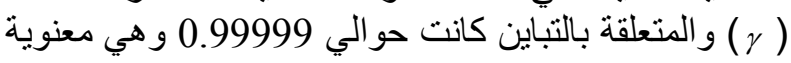

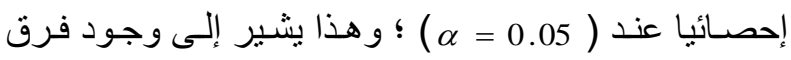

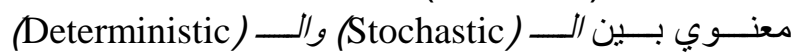
.Frontier

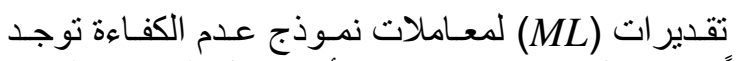

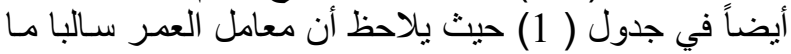

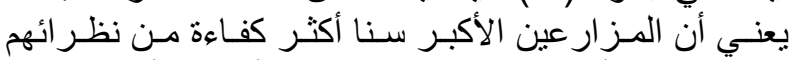

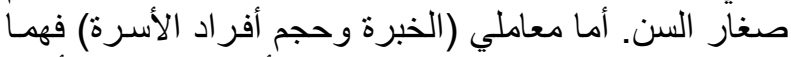

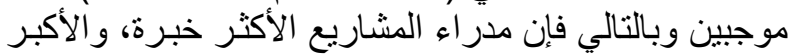

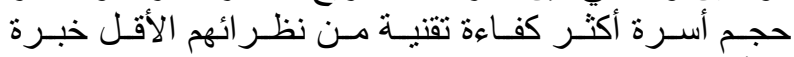

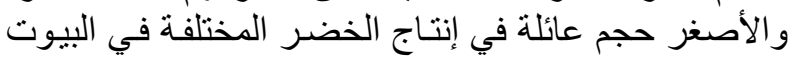
المحمية غير المكيفة.

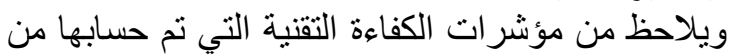

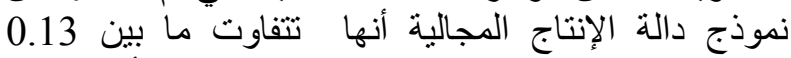

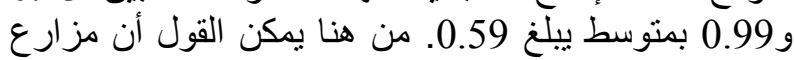

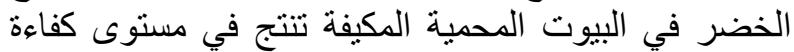

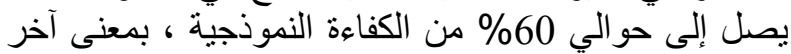

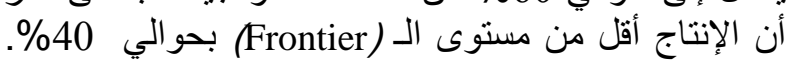

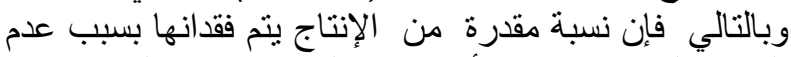

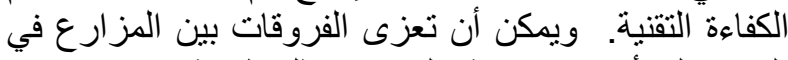

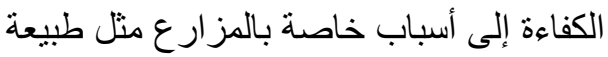

\section{3- مناقشة النتائج}

1-3-الكفاءة التقنية لمشاريع البيوت المحمية غير المكانج المكيفة

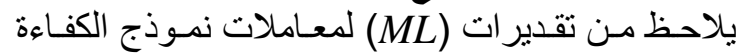

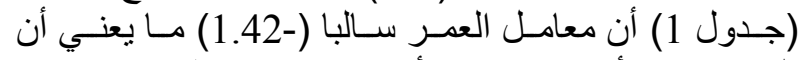

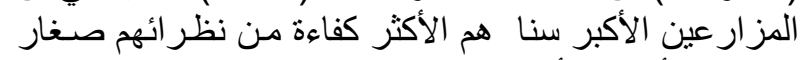

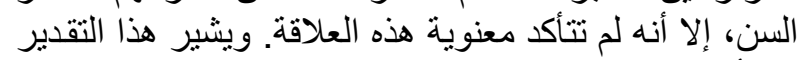

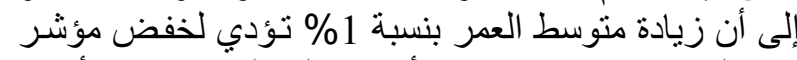

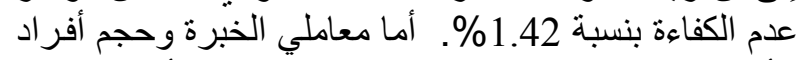

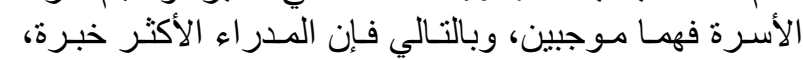

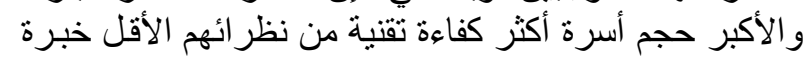

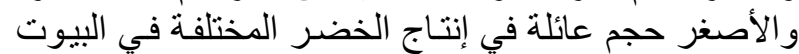
المحمية غير المكيفة. بينت نتائج تقدير المعاملات (جدول 2) أن المعاملات

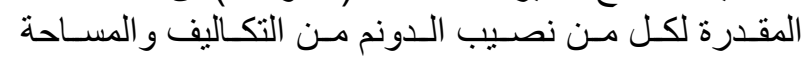

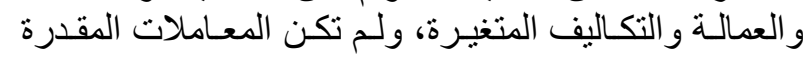

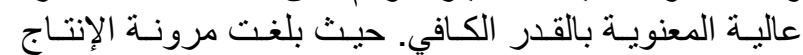

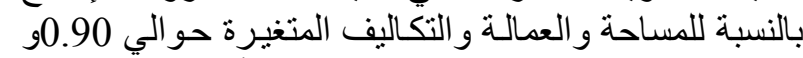

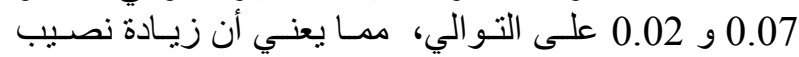

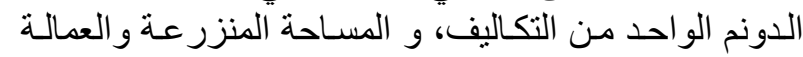

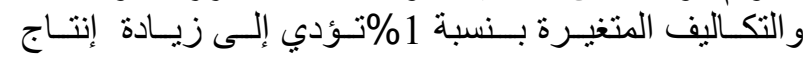

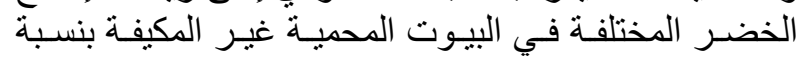

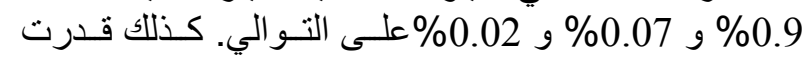

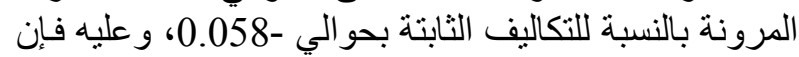

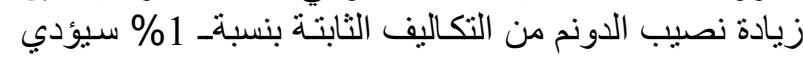

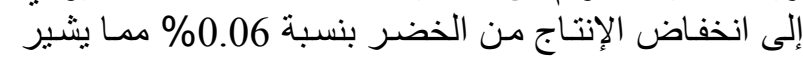

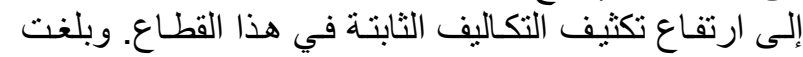

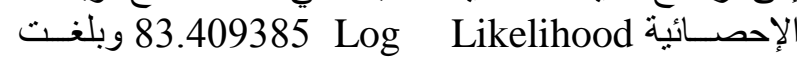
القيمة المقابلـة لـ 2.160940 OLS وبذا حسبت Log Ratio Liklihood

$(L R=-2(99.160940-83.409385)=31.5031)$

و هي غير معنوية، و عليه قبلت فرضية غيـاب الكفاءة التقتيـة

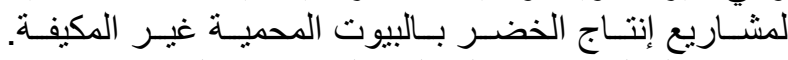
وقدرت المعلمة ( ر ) و المتعلقة بالتباين بحو الي 0.996068

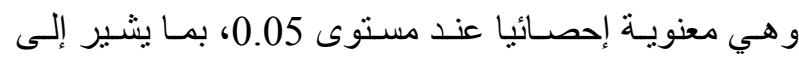
وجود فرق معنوي بين المجال العشوائي و المحدد.

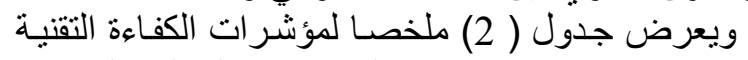

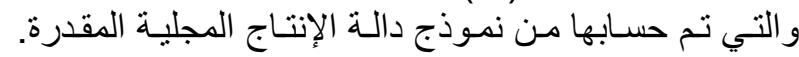

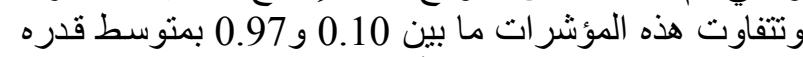

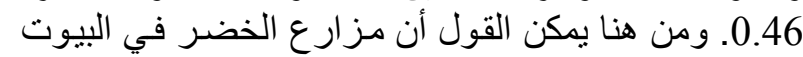

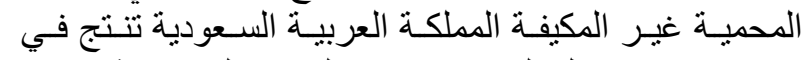

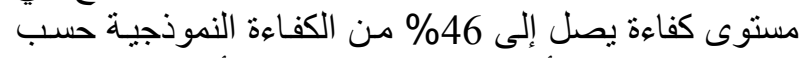

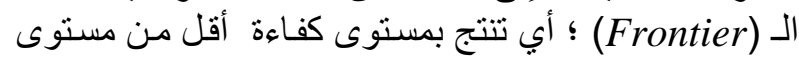

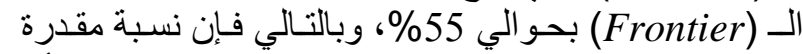

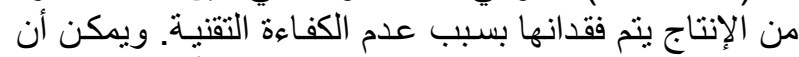

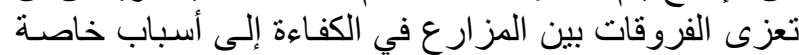

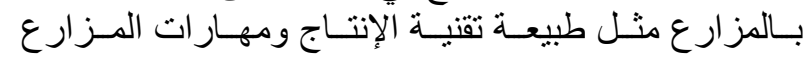
الإنتاجية (Ben-Belhassan,2000) 


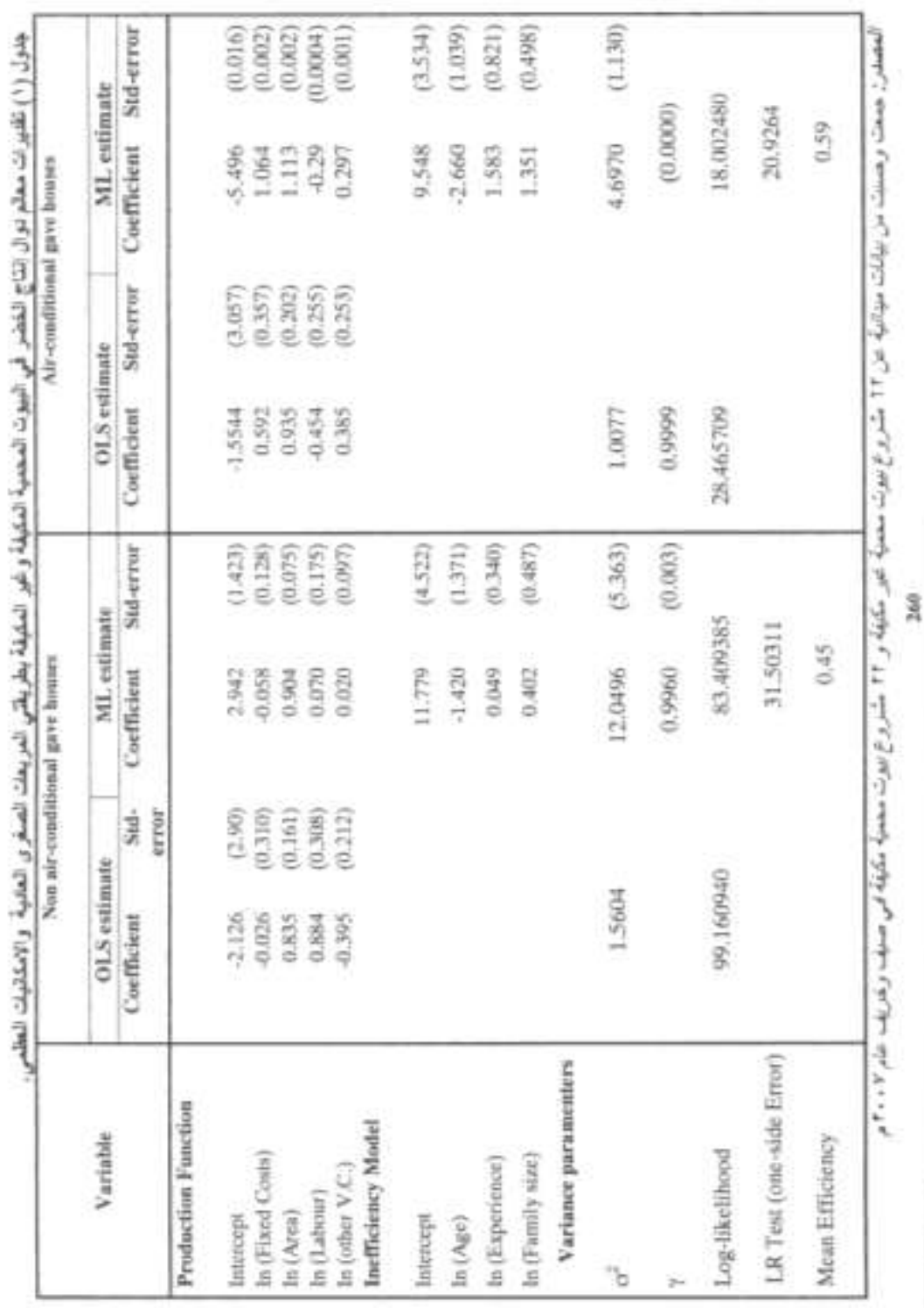




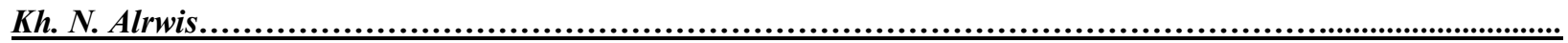

جدول (2) الكفاعة التقنية لإنتاج الخضر في البيوت المحمية غير المكيفة والمكيفة في المملكة العربية السعودية

\begin{tabular}{|c|c|c|c|c|c|c|c|}
\hline \multicolumn{7}{|c|}{ Non air-conditinal gave houses } & \multicolumn{3}{c|}{ Air-conditinal gave houses } \\
\hline firm firm & $\begin{array}{c}\text { Technical } \\
\text { Technical } \\
\text { Efficiency(TE) }\end{array}$ & firm & $\begin{array}{c}\text { Technical } \\
\text { Efficiency(TE) }\end{array}$ & firm & $\begin{array}{c}\text { Technical } \\
\text { Efficiency(TE) }\end{array}$ \\
\hline 1 & 0.28 & 22 & 0.91 & 43 & 0.26 & 1 & 0.59 \\
\hline 2 & 0.69 & 23 & 0.48 & 44 & 0.12 & 2 & 0.35 \\
\hline 3 & 0.23 & 24 & 0.02 & 45 & 0.87 & 3 & 0.30 \\
\hline 4 & 0.34 & 25 & 0.02 & 46 & 0.01 & 4 & 0.93 \\
\hline 5 & 0.01 & 26 & 0.50 & 47 & 0.45 & 5 & 1.00 \\
\hline 6 & 0.38 & 27 & 0.44 & 48 & 0.73 & 6 & 0.29 \\
\hline 7 & 0.84 & 28 & 0.61 & 49 & 0.85 & 7 & 0.44 \\
\hline 8 & 0.86 & 29 & 0.12 & 50 & 0.41 & 8 & 0.35 \\
\hline 9 & 0.83 & 30 & 0.44 & 51 & 0.01 & 9 & 0.01 \\
\hline 10 & 0.05 & 31 & 0.03 & 52 & 0.87 & 10 & 0.41 \\
\hline 11 & 0.62 & 32 & 0.43 & 53 & 0.07 & 11 & 0.76 \\
\hline 12 & 0.58 & 33 & 0.56 & 54 & 0.52 & 12 & 0.72 \\
\hline 13 & 0.62 & 34 & 0.59 & 55 & 0.74 & 13 & 0.66 \\
\hline 14 & 0.62 & 35 & 0.66 & 56 & 0.06 & 14 & 0.09 \\
\hline 15 & 0.57 & 36 & 0.56 & 57 & 0.60 & 15 & 1.00 \\
\hline 16 & 0.35 & 37 & 0.61 & 58 & 0.10 & 16 & 0.58 \\
\hline 17 & 0.43 & 38 & 0.58 & 59 & 0.78 & 17 & 0.29 \\
\hline 18 & 0.60 & 39 & 0.62 & 60 & 0.75 & 18 & 0.99 \\
\hline 19 & 0.59 & 40 & 0.17 & 61 & 0.70 & 19 & 0.41 \\
\hline 20 & 0.77 & 41 & 0.14 & 62 & 0.10 & 20 & 1.00 \\
\hline 21 & 0.56 & 42 & 0.17 & & & 21 & 0.88 \\
\hline & & & & & & 22 & 1.00 \\
\hline
\end{tabular}

المصدر: جمعت وحسبت من بيانات ميدانية عن 62 مشروع بيوت محمية غير مكيفة و 22 مشروع بيوت محمية مكيفة في صيف وخريف عام 2007م

جدول (3) معالم دالة الإنتاج والإمكانيات العظمى (MLE) لجميع مزارع الخضر غير المكيفة و المكيفة باستخدام الحزمة الاحصائية NLOGIT. و أنحدار العوامل الاجتماعية وفقا للتوزيع المبتور من طرفيه.

\begin{tabular}{|c|c|c|c|c|}
\hline \multirow[b]{2}{*}{ Variable } & \multicolumn{2}{|c|}{ Non air-conditinal gave houses } & \multicolumn{2}{|c|}{ Air-conditianed gave houses } \\
\hline & Coefficient & T test & Coefficient & T test \\
\hline Production function & & & & \\
\hline Intercept & & & 2.610 & $(1.859)$ \\
\hline $\ln$ (Fixed Costs) & 0.0013 & $(0.007)$ & 0.180 & $(1.480)$ \\
\hline $\ln ($ Area $)$ & 0.978 & $(9.994) *$ & 0.967 & $(6.890)^{*}$ \\
\hline $\ln$ (Labour) & 0.472 & $(1.760)^{* *}$ & -0.329 & $(-1.912 * *)$ \\
\hline $\ln$ (otherV.C..) & -0.129 & $(-0.626) \quad 0.184$ & (1.074) & \\
\hline Log Likelihood & 88.023 & & 43.503 & \\
\hline $\operatorname{Sigma}(u)$ & 1.84 & & 0.699 & \\
\hline Sigma(v) & 0.198 & & 0.283 & \\
\hline Lambda $\left(\lambda=\frac{\sigma_{u}}{\sigma_{v}}\right)$ & 9.327 & & 2.468 & \\
\hline Theta $\left(\theta=\frac{\lambda^{2}}{\lambda^{2}+1}\right)$ & 0.98 & & 0.86 & \\
\hline$E(-U)=\frac{1}{1+\theta}$ & & & & \\
\hline Exp. Efficiency $=\frac{1}{1+\theta}$ & 0.51 & & 0.54 & \\
\hline Tech. Inefficiency & 0.49 & & 0.46 & \\
\hline Tech. Efficiency Model & cated from bo & ides) & & \\
\hline $\begin{array}{l}\text { (Age) } \\
\text { Experience) } \\
\text { (Family size) } \\
(* *) \text { Denote t-statistic sig } \\
(*) \text { Denote t-statistic sig }\end{array}$ & $\begin{array}{c}0.0009 \\
0.397 \\
0.467 \\
\text { nce level of } 5 \% \\
\text { ice level of } 1 \%\end{array}$ & $\begin{array}{c}(2.254)^{*} \\
(5.642)^{*} \\
(0.479)\end{array}$ & $\begin{array}{c}0.125 \\
0.22 \\
-\quad 0.016\end{array}$ & $\begin{array}{l}(1.434) \\
(0.406) \\
(-0.302)\end{array}$ \\
\hline
\end{tabular}




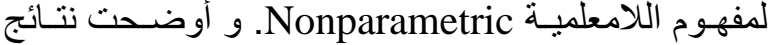

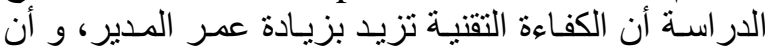

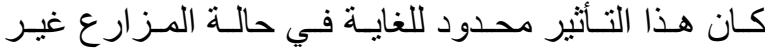

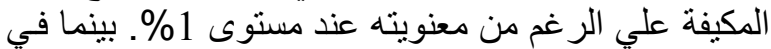

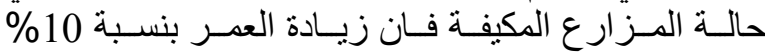

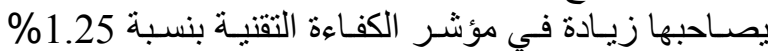

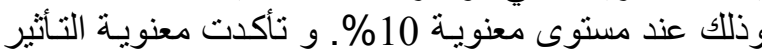

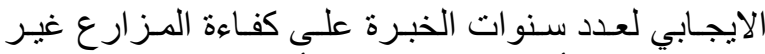

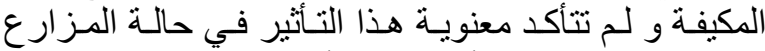

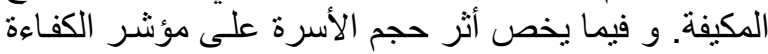

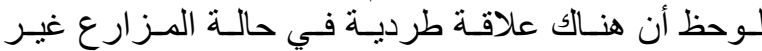
المكيفة و عكسية في حالة المز ارع المئة المكيفة و إن لم تتأكد معنوية العلاقة في الحالتين.

\section{الخلاصة و التوصيات}

من خلال تقدير الدالة المجالية العشوائية للبيوت المحمية

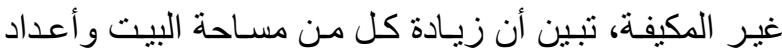
العمالة، و التكاليف المتغيرة بنسبة 10 \% \%، تؤدي إلى زئه زيادة

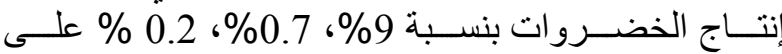
التو الي، في حين تؤدي زيادة التكاليف الثابتة بنسبة 10 \% \%

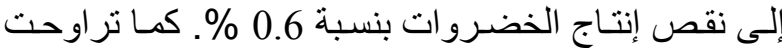

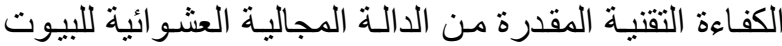

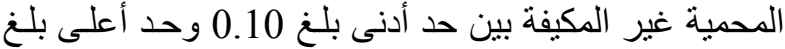

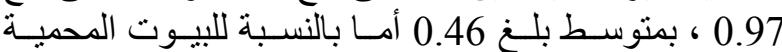
المكيفة، فقد تبين أن زيادة كل من التكاليف الثابتـة ومساحة

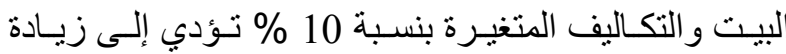

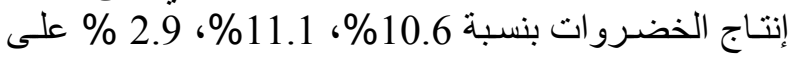

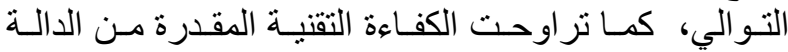

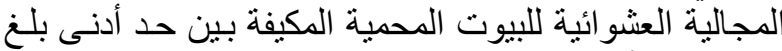

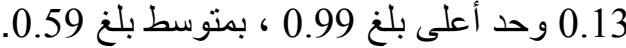

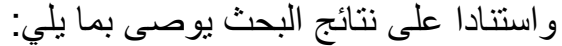

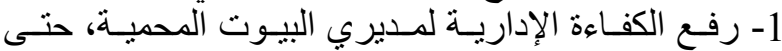

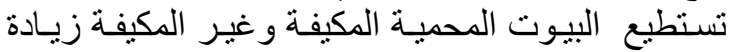

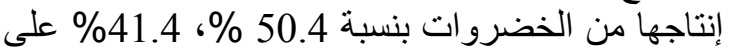
التو الي، دون أي زيادة في القدر المستخدم من الموارد الاقتصنادية.

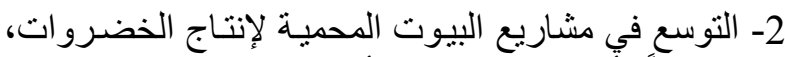

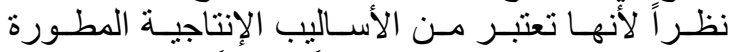

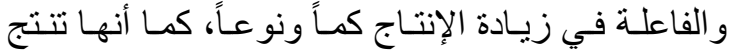

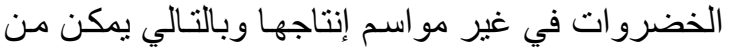

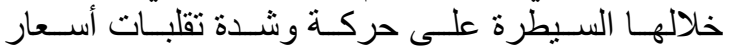

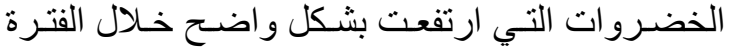

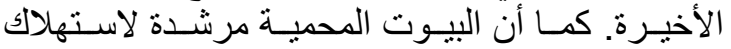

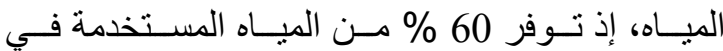
الزر اعة المكثوفة، وهذا بـت بتفق مع اعتبار ات الأمـن المائي في المملكة العربية السعودية.

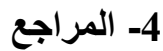

-1-1-مراجع باللغة العربية - وزارة الاقتصاد والتخطيط، إحصاءة التهات الناتج المحلي المحلي الإجمالي، بيانات غير منشورة، 2008م.
تقنيـة الإنتـاج ومهـار ات المـزارع الإنتاجيـة ـ ومـن خـلال

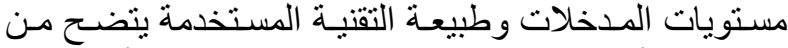

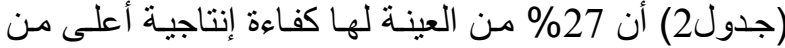

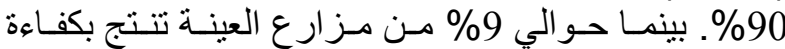

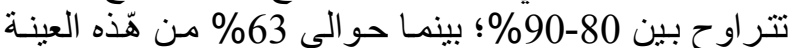
لها كفاءة تقنية تقل عن 80 80 \%

3-3- أثر بعض العضوامـل الاجتماعيـة على الكفـاءة التقتيـة

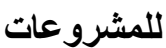

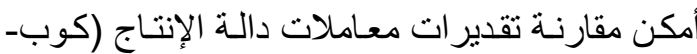

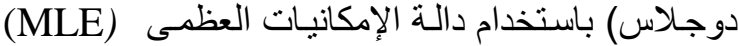

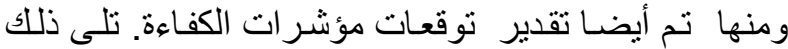

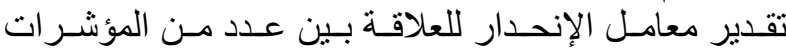
الإجتماعية و مؤشر الكفاءة التقنية، و الجديد في هذا التها التقدير

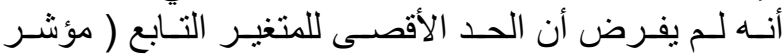

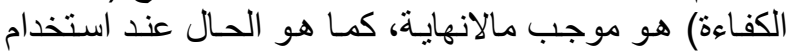

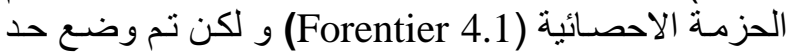

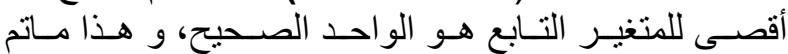

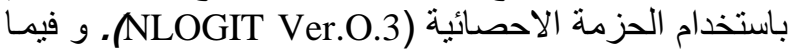
يلي سيتم عرض نتائج هذه التقدير ات كما بتضح من الجدول رقم (3). حيث لم تتأكد معنوية تأثير بند التكاليف الثابتة في التي التئي

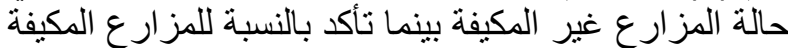

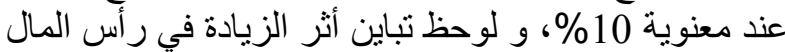

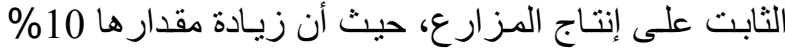

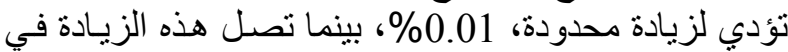

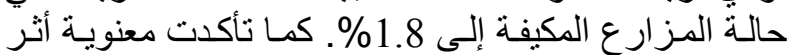

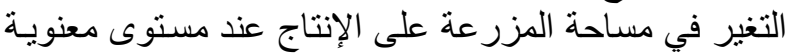
1\%، و تقاربت كلا النسبتين فكانتا 9.8\% و و و 9.7 9.

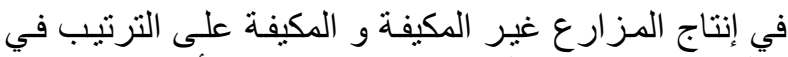

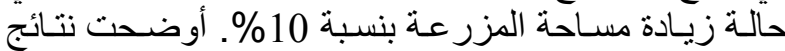

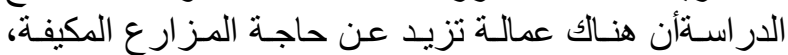

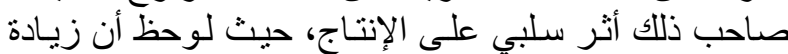

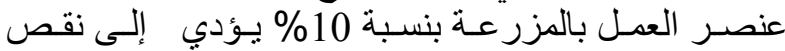

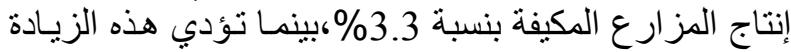
في عنصر العمر في المزارع غير المكيفة إلى زيادة الإنتاج

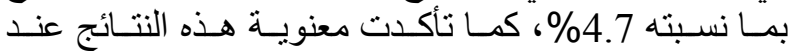

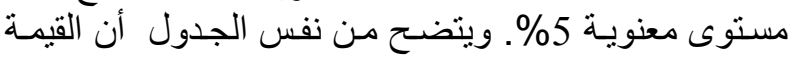

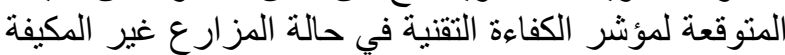

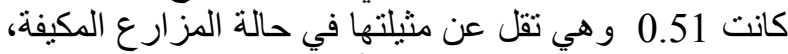

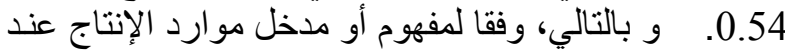

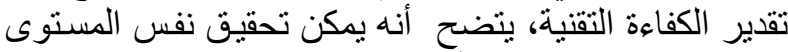

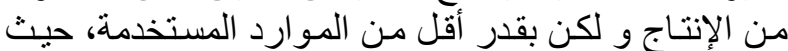

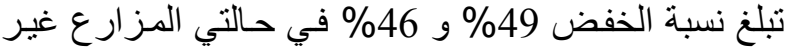
المكيفة و المكيفة على الترتيب.

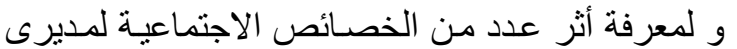

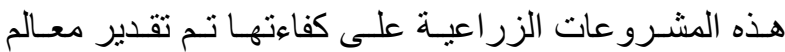

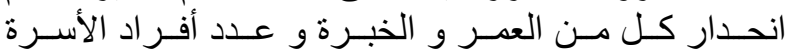

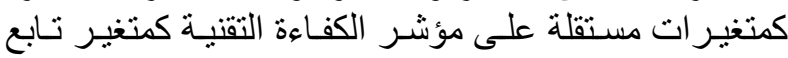

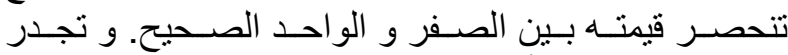

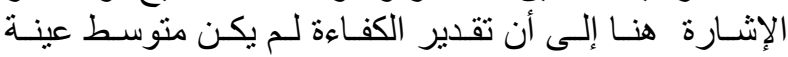
الدراسة بل نم تقدير هذا المؤشر لكل مزر عة على لـد حدة وفقا 


$$
\begin{aligned}
& \text { - وزارة الزراعة، الكتاب الإحصائي الزراعي السنوي، }
\end{aligned}
$$

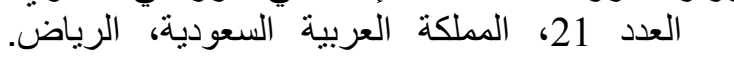

$$
\begin{aligned}
& \text { 2008م. }
\end{aligned}
$$

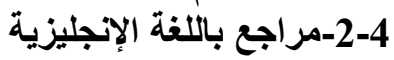

Afriat S.N.(1972). Efficiency estimation of production functions. Inter. Econ. Rev. 13: 568-98.

Aigner D.J., Lovell C. A. K. and Schmidt P. (1977). Formulation and estimation of stochastic frontier production function models. J. of Econometrics 6: 21-37.

Battese G.E. (1998). A stochastic frontier model for the analysis of the effects of quality of irrigation water on crop yields. (CEPA) Working Paper 98/09, Department of Econometrics, University of New England, Armidale, Australia.

Ben-Belhassen B. (2000). Measurement and explanation of technical efficiency in Missouri hog production. elected paper, AAEA meetings, Tampa City, Florida.
- وزارة الزراعة، الكتاب الإحصائي الزراعي السنوي ،

العدد 207، الزملكة العربية السعودية، الرياضي الرئي

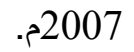

Coelli T.J. (1996). A guide to FRONTIER Version 4.1: A computer program for frontier production function estimation. (CEP) Working Paper 96/07, Department of Econometrics, University of New England, Armidale, Australia.

Coelli T.J., D.S., Prasada R., and Battese G. E. (1998). An Introduction to Efficiency and Productivity Analysis Kluwer Academic Publishers,London,UK.

Greene W.H. (2003) NLOGIT 3.0, Econometric Software, USA.

Maddala G.S. (1987). Limited Dependent and Qualitative Variables in Economics. Cambridge University Press, New York 